$\begin{array}{ll}\text { Italique } & \text { Italique } \\ \text { Poésie italienne de la Renaissance }\end{array}$

XIX | 2016

Varia

\title{
«Sorgi Homer, vien Petrarca, esci Marone». I corrispondenti in versi di Luigi Groto
}

\section{Barbara Spaggiari}

\section{(c) OpenEdition}

\section{Journals}

\section{Edizione digitale}

URL: http://journals.openedition.org/italique/438

DOI: $10.4000 /$ italique.438

ISSN: 1663-4438

\section{Editore}

Librairie Droz

\section{Edizione cartacea}

Data di pubblicazione: 1 dicembre 2016

Paginazione: 247-262

ISBN: 978-2-600-04777-7

ISSN: 1423-3983

Notizia bibliografica digitale

Barbara Spaggiari, « «Sorgi Homer, vien Petrarca, esci Marone». I corrispondenti in versi di Luigi Groto », Italique [Online], XIX | 2016, online dal 01 décembre 2018, consultato il 07 septembre 2019. URL: http://journals.openedition.org/italique/438; DOI : 10.4000/italique.438

(C) Tous droits réservés 
B A R B A A S PAGGIAR I

«S ORG I H O ER, VIEN PETRARCA, E S C I M A R O N E».

I CORRISPONDENTI IN VERSI

D I L UIG I GROTO 



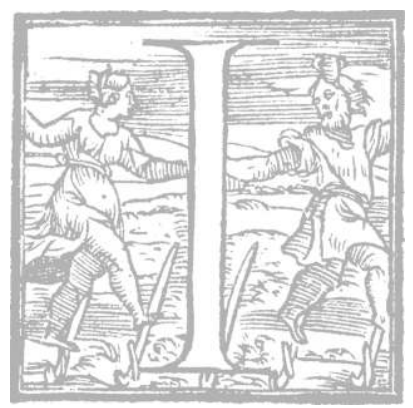

l genere delle rime di corrispondenza, inteso come scambio epistolare in versi che contempla una proposta e una risposta, occupa una zona assolutamente marginale nella produzione lirica di Luigi Groto, e del resto non figura nella Prima parte delle Rime, la sola curata dall'autore per la stampa (I577, I584').

L'ultimo editore secentesco, Ambrogio Dei, che si prefisse lo scopo di riunire la totalità delle poesie attribuite al Cieco d'Adria, recuperò appena un paio di testi oggetto di scambio fra il Groto e il Bonardo, entrambi pubblicati già nel I563. ${ }^{\mathrm{I}}$ Anche in questo caso, e malgrado il rapporto molto stretto che esisteva fra $i$ due corrispondenti, a prendere l'iniziativa non fu il Groto, bensi il Bonardo. Secondo una costante destinata a confermarsi nel prosieguo, il Cieco accettava talvolta di rispondere in versi alle sollecitazioni altrui; però non gli si conoscono sonetti (o altre forme poetiche) propriamente 'di invio', che abbiano dato luogo a un ritorno da parte del destinatario. ${ }^{2}$

Le poche e non certo memorabili rime di corrispondenza, reperite nel corso dei lavori preparatori alla recente edizione dell'opera, trovano spazio nel paratesto di alcuni volumi di cui il Groto fu curatore; oppure, sono messe in exergo da altri autori al principio della propria raccolta, al fine di assicurarsi la presenza benevola di un nome famoso. L'idiosincrasia del Cieco nei confronti di questa tradizione secolare, dalla quale palesemente rifugge, trova probabilmente giustificazione nel fatto ch'egli stesso ba curato una propria raccolta di lettere, scritte però in prosa e non in versi, con la quale mostra in modo inequivoco di volersi profilare come modello di epistolografia per i contemporanei. Le sue Lettere famigliari, già nella scelta ciceroniana del titolo, pretendono infatti di fornire una serie di campioni paradigmatici per chi intenda scrivere una missiva non solo adeguata a singole circostanze ma, soprattutto, rispettosa dei canoni stilistici che presiedono a tale genere letterario. Queste lettere sono normalmente precedute da un'etichetta d'uso, per indicarne il tono o lo scopo specifico. ${ }^{3}$

Oratore insigne, Groto amava far sfoggio delle proprie doti retoriche anche nell'ambito epistolare. Invece, nella corrispondenza in versi, la sua vena poetica di solito fluente, e a tratti debordante, sembra inaridirsi 
in una serie di stucchevoli elogi e di banalità d'occasione. Più che $i$ contenuti o la forma, sono appena $i$ nomi dei corrispondenti che suscitano un qualche interesse, in quanto permettono di ricostruire una rete di rapporti, che in questo caso è giusto designare come eminentemente provinciale. $^{4}$

Non sono i grandi intellettuali dell'epoca che scambiano rime con il Cieco d'Adria: sono, piuttosto, i velleitari amanti delle belle lettere che, dal loro oscuro scranno in qualcuna delle numerose accademie tardocinquecentesche disseminate nelle città del nord-est, fra le Venezie e il Friuli, si avventurano a chiedere la malleveria di colui che era pur sempre un rispettato Principe (degli Illustrati in Adria), e un membro illustre di Accademie ben più prestigiose (gli Olimpici di Vicenza, $i$ Concordi di Rovigo, i Pastori di Fratta).

Fa eccezione, a suo modo, proprio il già menzionato Giovan Maria Bonardo, conte della Fratta di Polesine, fondatore dell'omonima Accademia dei Pastori frattegiani, che apriva le sue porte a personaggi del calibro di Girolamo Ruscelli, Lodovico Dolce, Girolamo Parabosco, Lodovico Domenichi, i conti Tiene, Giacomo Tiepolo, Ercole Udine, Orazio Toscanella, Adriano Carignano, Antonio Beffa Negrini. Autore di più volumi, alcuni ristampati per decenni, il Bonardo ricorse in modo sistematico alle competenze del Groto in funzione (si direbbe oggi) di editor. Non meraviglia, dunque, che gli renda omaggio con un testo liminare dei Madrigali.

Il Bonardo, ${ }^{5}$ oltre che alla letteratura da intrattenimento, si dedicava ben più seriamente alla trattatistica, ricorrendo a un tipo di prosa a metà fra la dissertazione scientifica e il manuale d'uso immediato. La sua opera più famosa, Le ricchezze dell'agricoltura, ${ }^{6}$ si inserisce in un ricco filone di testi agronomici che mettono in risalto le delizie e le vaghezze della vita "in villa", lodando al tempo stesso i prodotti dovuti a un impiego attento e consapevole della coltivazione. ${ }^{7}$ Accanto ai classici più volte volgarizzati, dalle Georgicae di Virgilio alla raccolta De re rustica (che include gli scritti di Catone il vecchio, Varrone, Columella e Palladio), in questa epoca si moltiplicano le nuove opere sia di livello letterario - La coltivazione dell' Alamanni e Le Api del Rucellai - che utilitaristico, con i sempre "notevoli" ammaestramenti di agronomi avvertiti. ${ }^{8}$

L'opera di Andrea Nicolio - altro corrispondente del Groto - fa invece parte della «folta e, talvolta, dignitosa storiografia municipale»," che 
durante la seconda metà del secolo e gli inizi del Seicento si adoperò assiduamente a nobilitare le origini di ogni città, tracciandone poi gli eventi principali secondo uno schema fisso: dopo il racconto della fondazione, sempre leggendaria ed illustre, si accenna al periodo romano $e$ all'oscura epoca medievale, per poi lodare il dominio della Serenissima, che porta finalmente pace e benessere a tutti $i$ devoti sudditi. L'omaggio protocollare a Venezia non sempre riesce a nascondere del tutto il desiderio di una maggiore autonomia; senza contare l'orgoglio cittadino, che fa di ogni antico comune l'ombelico del mondo.

La Historia di Rovigo di Andrea Nicolio ${ }^{\mathrm{IO}}$ rispetta questa tacita tendenza, tanto che il ruolo di primo piano da lui riservato ai Rodigini nella storia del Polesine suscitò vive polemiche. Rovigo, «ricco, \& opulento», con la sua Accademia dei Concordi, di cui il Groto era membro, suscitava - allora come oggi - la rivalità di Lendinara e di Adria. I termini della questione sono dibattuti in una curiosa Apologia [...] sopra l'Historia del Sig. Andrea Nicolio, ${ }^{\text {II }}$ composta da Ercole Cato $^{\mathrm{I}}$ e dedicata "AL MOLTO ILLUSTRE ET REVERENDISSIMO MONS. GIVLIO CANANI Vescovo d'Adria». In forma di dialogo fra due sostenitori delle opposte fazioni, l'Apologia riporta in dettaglio le accuse mosse al Nicolio, ma in realtà a Rovigo, cui viene contestato perfino il titolo di 'città', perché non possiede una cattedrale e non è sede vescovile, a differenza di Adria, da sempre a capo di un'importante diocesi. ${ }^{{ }^{3}}$ L'operetta del Cato è un tipico esempio dello spirito fondamentalmente municipale legato al proliferare di Accademie di belle lettere o, più raramente, di scienze in quasi tutte le località del Veneto. ${ }^{14}$

Fra dispute campanilistiche e problemi relativi alla gestione delle terre e dei fumi, si disegnano i contorni dell'ambiente in cui vive ed opera Luigi Groto, che è nomo di terraferma come il Bonardo e il Nicolio: nato fra le paludi del delta, è toccato in prima persona dalle piene del Po, che lo privano precocemente di quel poco di eredità terriera lasciatagli dal padre. I suoi orizzonti e $i$ suoi interessi sono vicini alla piccola nobiltà fondiaria, ai padroni delle ville che accolgono generosamente gli intellettuali squattrinati come lui, nell'unica forma di mecenatismo puramente alimentare - che sopravvive alla scomparsa del modello del cortigiano. Venezia e la sua tradizione marinara, l'aristocrazia della Serenissima, i circoli degli intellettuali che ne assicurano il predominio culturale, la presenza degli editori in assoluto più prestigiosi: tutto questo è lontano dal mondo del Groto, e di difficile accesso. 
Certo, Groto svolse la funzione di ambasciatore in nome di Adria; ebbe ripetuti contatti con Domenico Venier; collaborò a più riprese con Lodovico Dolce e Girolamo Ruscelli; fu amico della Gasparina Pitoni, autrice del suo ritratto xilografico; stampò tutte le sue opere nella tipografia degli Zoppini, bottega di solido prestigio. Ma nonostante questi continui rapporti e la multitudine dei suoi legami, Groto ottiene il passaporto veneziano solo sul finire della vita, e senza nemmeno riuscire a goderselo, perché la morte lo coglie prima che prenda possesso della cattedra di filosofia nella Scuola di Rialto. Alle sue radici polesane lo riconduce quasi a forza un altro interlocutore, forse il più noto dei pochi suoi corrispondenti, quel Giovan Battista Maganza che, celandosi dietro lo pseudonimo di Magagnò, organizzò una celebre raccolta di rime in dialetto pavano. ${ }^{15}$ Durante la preparazione della quarta ed ultima parte di queste Rime, il Maganza invia un 'sonagetto' ad Alvise Grotto, che in quel momento si trova ospite in un' altra delle ville venete, l'Eolia di Costozza, proprietà del conte FrancescoTrento. Anche qui un'accademia, anche qui un cenacolo di dotti letterati, fra iquali le duepoetesse Issicratea Monti e Maddalena Campiglia. ${ }^{16}$

Groto, che pur non ama verseggiare in dialetto, non può esimersi dal rispondere all'invito del Maganza, e lo fa per le rime, ovviamente in lingua rustica, con un'abilità e un'arguzia che non sorprende in un giocoliere della parola come lui era. Però, anche le incursioni nell' ambito della poesia pavana restano numerate e, alla fine, risultano non piu che episodiche nell'ingente corpus delle rime grotiane. ${ }^{17}$

Esauriti i nomi di qualche notorietà, gli altri corrispondenti in versi del Cieco d'Adria appartengono alla massa dei letterati minori, se non minimi, del Cinquecento. Provengono da famiglie di medici, giuristi, notai, sono ricchi e quasi sempre nobili, si dedicano alla poesia come a un dovere sociale. Nella loro ricerca di una qualche visibilità, brigano per ottenere la partecipaz̧ione, direi quasi la complicità, di un poeta rinomato, la cui sola presenza era suscettibile di far uscire dal grigiore anonimo un libro di rime.

In questa categoria rientra di diritto don Lorenzo, monaco Cassinese, al secolo Pietro Massolo, nobile veneziano il quale, nel I564, pubblicò a Firenze una raccolta di ben ottocento sonetti morali, che occupano le prime 4 I8 pagine di un volumetto in ottavo. ${ }^{18}$ Le carte residue ospitano una cospicua serie di poesie laudatorie, inviate al Massolo da vari contemporanei, fra cui il Groto, con uno scambio per le rime. ${ }^{\text {I9 }}$ 
Il Massolo, che si proclama amico del Bembo, scriveva da tempo poesie di tema rigorosamente morale, intonate al suo nuovo statuto monacale ("dico, che'ldilettarè cosa accessoria; ma ilgiovarènecessario», op. cit., f. ${ }^{*}$ rr). La raccolta iniziale di appena 400 sonetti, stampata nel 1557 e dedicata al cardinale Alessandro Farnese, è un flagrante esempio di autoincensamento:

[ $\left.{ }^{*} \mathrm{r}\right]$ Tosto che io hebbi fornite le due mie opere in prosa latina, l'una della concordia di Platone, \& d'Aristotele, l'altra del modo, \& della ragione del comporre l'historia; le quali due mie opere essendo state già in vita approbate dal gran Bembo, spero in breve di mandar in luce; ${ }^{20} \mathrm{mi}$ diedi alle volte per mio trastullo a cantar in verso [...] \& havendo posto l'ultima mano al presente volume di rime, qual è de quattrocento sonetti; [...] [ $\left.{ }^{*} \mathrm{IV}\right]$ mi è paruto da questa mia opera, come da quella, che è tra tutte l'altre mie opere, \& per la qualità, \& per la varietà del sogetto più vaga, \& più dilettevole, \& più giovevole, $\&$ più profittevole a gli studiosi di buone lettere: incomminciar a dar qualche saggio di me, \& delle fatiche mie al mondo. (expl. «Di Santo Benedetto Mantovano: Il dì primo di Ottobre; nel MDLVI») $)^{21}$

Ben più che per la qualità delle rime, il volumetto è prezioso per ricostruire la rete di personaggi con cui il Massolo era (o desiderava essere) in contatto; nelle epigrafi, fra $i$ destinatari dei sonetti, ritroviamo Annibal Caro, Sperone Speroni, Vittoria Colonna, Bernardo Capello, Lodovico Dolce, Anton Francesco Doni, Alessandro Piccolomini, Bernardo Tasso, Francesco Sansovino, Girolamo Ruscelli, Lorenzo e Luigi Priuli, Luigi Alemanni, Giovan Giorgio Trissino, Ercole e Guglielmo Gonzaga, Giovanni Soranzo, Ludovico Castelvetro, Domenico Venier e Benedetto Varchi. ${ }^{22}$

Gli ultimi scambi di corrispondenza poetica del Groto risalgono al I583 e si inseriscono in un quadro totalmente diverso. Sollecitato da due differenti interlocutori, il Groto riceve l'invito a partecipare ad una miscellanea bilingue in omaggio del re di Polonia, Stefano Battori (Báthory). ${ }^{23}$ L'iniziativa di stampare questa raccolta viene talora attribuita, per errore, al conte Giacomo Zabarella, accademico degli Elevati in Padova; però né il suo, né altro nome di curatore appare nei frontespizi dei due volumi, che accolgono rispettivamente $i$ carmi latini e le rime in volgare scritti in onore del Principe di Transilvania.

Studi recenti hanno permesso di individuare in Modesta da Pozzo de' Zorzi (I555-I592), alias Moderata Fonte, la regista silenziosa di questa 
operazione, al tempo stesso culturale e religiosa, che fin dal titolo sceglie una tonalità media: Viridarium e Giardino prendono il posto di templi, ghirlande, corone e altri altisonanti attributi che di solito ornano simili antologie.

Il ruolo di Moderata Fonte nell'organizzare il doppio volume si rivela in almeno due aspetti: non solo il suo è l'unico nome di donna che figura nella lista dei quaranta autori (Giardino, $f .{ }^{*}$ Ir $r$, ma è quello che più di ogni altro ha contribuito, con le proprie poesie, ad arricchire la raccolta celebrativa. E superfluo ricordare che questa scrittrice veneziana, considerata una proto-femminista e come tale riscoperta a partire dagli anni'so del secolo scorso, era perfettamente in grado di comporre in latino, ${ }^{24}$ grazie all' educazione ricevuta in casa, prima per le cure del nonno Prospero Saraceno, poi dello zio acquisito, Giovanni Niccolò Doglioni. ${ }^{25}$

L'ambiente friulano, cui appartenne anche Irene Spilimbergo, altra figura di spicco della cultura 'al femminile' verso la metà del Cinquecento, ${ }^{26}$ fornisce un buon numero di collaboratori alla miscellanea in onore del re polacco: fra questi, Gervasio Gervasi ed Antonio Cavassico (Cavassego), che appunto invitarono il Groto, indipendentemente l'uno dall'altro, a prendere parte all'omaggio collettivo. ${ }^{27}$

Gervasio Gervasi è citato dal Miari con una breve scheda: "GERVASIS Gervasio. Compose un'opera eroica in lode di Sigismondo Battori, principe di Transilvania, ${ }^{28}$ ed inoltre alcune orazioni, elegie ed un'ode al S. Nicolo di Bari. Mori quasi ottuagenario il 21 novembre I6r4〉. Possiamo aggiungere che fu membro dell'Accademia Colle Bellunese, fondata dopo la metà del secolo dal medico Giovanni Colle perché $i$ letterati locali avessero «un luogo di comune esercizio de' loro talenti». ${ }^{29}$ Diversamente, il Cavassico appartiene a una delle dinastie bellunesi più in vista; fino dal secolo precedente, la sua era una famiglia di notai e giuristi, con velleità letterarie. ${ }^{30}$ La tenue fama di Antonio è legata ad un'unica opera, L'Aprile (In Trevigi: presso Angelo Mazzolini, I590), liquidata con poche righe nel catalogo del Pagani: «E una raccolta di componimenti poetici in metro vario, che dedicò a Pietro e Nicolò Dolfin, figli del veneto patrizio Luigi. Prima dei versi trovasi una Orazione». ${ }^{31}$ L'unico esemplare censito di quest'opera si trova in una miscellanea della Biblioteca Nazionale Marciana (Misc. 2450) che contiene altre cinque raccolte di versi, stampate fra il I548 e il I599: le Rime di Lodovico Domenichi, ${ }^{22}$ Le fiamme di Giraldi Cinzio, ${ }^{33}$ un 
prosimetro senza indicazione di autore, ${ }^{34} \mathrm{La}$ Psiche di Ercole Udine $^{35}$ e un Lamento anch'esso anonimo. ${ }^{36}$

$\mathrm{Nel}$ registro medio, e direi quasi sotto tono, che connota questa miscellanea marciana, L'Aprile del Cavassico si presenta con le caratteristiche di un volume estremamente curato, con carta di ottima qualità, caratteri e xilografie nitide; una cornice fiorita include il testo di ogni pagina. Tutte le autorità di Belluno, Treviso e Cividale sono oggetto di riverente omaggio da parte dell'autore, che qui raccoglie, con tutta evidenza, il meglio della propria produzione in versi, sia volgari che latini. ${ }^{37}$ I sonetti al re di Polonia e quello «a M. Luigi Grotto con risposta» occupano il posto d'onore, rispettivamente ai ff. Igv-20v e 2Ir-24v. Curiosamente, il Cavassico usa lo stesso pseudonimo Damon(e) che il Cieco portava come accademico della Fratta, ${ }^{38}$ e il nome della città di Adria compare almeno due volte nella raccolta, ai ff. $25 r$ e 4 Ir.

I sonetti con cui Groto risponde all'invito dei due letterati friulani non lasciano trasparire entusiasmi particolari, né brillano per originalità. Sono di fatto il risultato di un riciclaggio, che prende per base un dittico composto in onore di Barbara d'Austria, seconda moglie di Alfonso II d'Este (ante quem I572), ripescato poi dal Dei e inserito nella Seconda parte delle Rime (II.Is6 e Is7).

Perché venga richiesto al Groto di contribuire al Viridarium / Giardino, è del resto intuibile. Una decina d'anni prima egli si era fatto carico, come organizatore, di quel Trofeo per la vittoria di Lepanto che si aggiunge alle altre innumerevoli acclamazioni della storica sconfitta dei Turchi (7 ottobre I57I), usando lo stesso modello articolato sui versanti paralleli del latino e del volgare (nella fattispecie, pluridialettale). Il paragone fra $i$ due eventi può sembrare improponibile, e lo è, senza dubbio, tenuto conto delle forze in campo e delle conseguenze geo-politiche. Resta però l'identità degli scopi: gli autori della miscellanea bilingue vedono in Stefano Báthory un paladino del cattolicesimo, da lui imposto in una Polonia multietnica con una copiosa varietà di credi religiosi. ${ }^{39}$ Il suo tentativo di creare una nuova lega cristiana in funzione anti-turca, cioè un baluardo contro le orde musulmane che premevano ai confini della cristianità, era però destinato all'insuccesso.

A titolo di curiosità, sia lecito sottolineare che, proprio nel Trofeo per la vittoria di Lepanto organizzato dal Groto, figurano rime di altre 
due poetesse venete, Giannetta Tron ${ }^{40}$ e Rosa Levi, ${ }^{4 \mathrm{I}}$ «ombre di letterate» (la definizione è di Daria Martelli) che lasciarono solo lievi tracce nella lirica tardo-cinquecentesca. È comunque sorprendente che fra i Iso componimenti che costituiscono il Trofeo - un numero tutto sommato modesto per le consuetudini dell' epoca - trovino diritto di cittadinanza due esponenti della cultura 'al femminile', contemporanee di Moderata Fonte. La fama di misogino, che Groto si guadagnò con la lettera in prosa dello pseudo-Lyndorach ${ }^{42}$ e con il divertente capitolo in terzine sul 'toglier moglie', 43 si rivela probabilmente ingiusta.

Del resto, la corrente - secolare e mai esausta - degli scritti contro le donne o delle speculazioni sui loro difetti, forniva al Groto almeno due esempi a lui prossimi: il dotto trattato latino di Giovanni Della Casa, Quæstio lepidissima an uxor sit ducenda; ${ }^{44}$ e la satira quinta di Ludovico Ariosto, che con particolare brio esorta un suo parente a rinunciare alle nozze già programmate. ${ }^{45}$ La questione meriterebbe di essere approfondita, ma fin d'ora possiamo escludere la generica etichetta di 'misoginia' affibbiata al Groto, e ricondurne l'atteggiamento ad una pura e semplice ricusa dell'istituto del matrimonio che, tralasciando $i$ due illustri precedenti letterari, trova la sua giustificazione teologica nel dibattito sviluppatosi, a partire da Lutero ed Erasmo, intorno alla sacralità o sacramentalità di tale istituzione.

Barbara Spaggiari 
I. Cfr. Madrigali del cavalier Gio. Maria Bonardo fratteggiano, In Vinegia: appresso Domenico Farri, I 563. A f. 46r Vorrei come ho il desio (Al Signor Luigi Groto Cieco d'Adria...) con la risposta del Cieco a f. 46v Quando haveste desio; a f. $46 \mathrm{v}$ Il dotto, vago, ameno, e dolce stile con la relativa risposta a f. $47 \mathrm{r}$ Mentre il mio ferreo, col vostro aureo stile. Per i testi e ogni altra citazione si rinvia a Le Rime di Luigi Groto, Cieco d'Adria. Edizione critica a cura di B. Spaggiari, 2 vol., Adria: Apogeo, 2014 (d'ora in poi Le Rime): vol. I, pp. cxv-cxviii.

2. Si escludono dall'analisi i rari esempi di dialogo fittizio, quali l'apostrofe al fiume Scortico (Rime I. I 5 , edita sempre nei Madrigali del Bonardo, a f. *8) o le due coppie di Proposta/Risposta che figurano nella Seconda Parte delle Rime (II.348-49 e 598-99).

3. Il volume è d'altronde fornito di una dettagliata Tavola dei generi delle lettere, dove si registrano anche le sfumature all'interno di un genere: ad es. «Nontiatoria» prevede ben sette sottogeneri, tra cui «Nontiatoria, e petitoria», «N., e laudatoria», «N., ò di Ragguagli».

4. A differenza dell'opera complessiva del Groto, il cui raggio di diffusione toccò vari paesi d'Europa (Francia e Inghilterra, Spagna e Portogallo, Austro-Bavaria, Croazia, Creta). Cfr. da ultimo B. Spaggiari, «Un nuovo tassello per la ricezione europea di Luigi Groto», in REM VI, 2 (20I 5), pp. 27-30.

5. Sul Bonardo esistono una monografia e altri studi anche recenti: cfr. S. Malavasi, Giovanni Maria Bonardo, agronomo polesano del Cinquecento, Venezia: Diputazione per la Storia Patria, I988; Ead., «Ancora sull'agronomo Giovanni Maria Bonardo e l'Accademia dei Pastori Frattegiani», in Verso la santa agricolutura. Alvise Cornaro, Ruzante, il Polesine, a c. di G. Benzoni, Rovigo: Minelliana, 2004, pp. 67-79.

6. In Venetia, Presso Fabio, \& Agostino Zoppini, fratelli, I 584, con numerose ristampe.

7. Anche dal punto di vista socio-politico, l'attività agricola aveva conquistato ormai il rispetto dei cittadini, e di Venezia in particolare, perché forniva alla repubblica marinara l'entroterra di cui necessitava per approvvigionarsi di "vittuaria". In quest'ottica vanno considerate anche le successive opere di bonifica e gli interventi sul delta per assicurare sempre più estesi luoghi atti a coltura.

8. A questo gruppo appartengono i contemporanei Agostino Gallo (Le dieci giornate della vera agricoltura, e piaceri della villa, i 564) e Camillo Tarello (Ricordo d'agricoltura, i 567 ). Che i trattati di agronomia siano fra i best-sellers dell'epoca lo dimostra anche la traduzione che Ercole Cato fece di un celebre manuale francese (cfr. qui, nota I 2 ).

9. Cfr. Storici e politici veneti del Cinquecento e del Seicento, a c. di G. Benzoni e T. Zanato, Milano-Napoli: Ricciardi, I982, p. xx.

Io. Cfr. Historia del Signor Andrea Nicolio, Dell'origine et antichità di Rovigo, con tutte le guerre, \& avvenimenti notabili fin' all' Anno MDLXXVIII, In Brescia: appresso Vincenzo Sabbio, i 578. Cfr. Le Rime, vol. I, pp. cxxxiv-cxxxv. 
I I. Il titolo completo è APOLOGIA | DEL ZAGO | DI SANTA | RENTVA, | sopra l'Historia del Sig. | Andrea Nicolio Dottor | da Rovigo, dell' Origine, | \& antichità di | quello.|| In Bologna | Per Gio. Rossi. MdlxxxiII.

I 2. Di origine ferrarese, dal $\mathrm{I}_{5} 63$ Ercole Cato svolse le funzioni di segretario presso il cardinale Ippolito II d'Este, fino alla morte di questi (I 572 ). Nominato cavaliere dal doge Sebastiano Venier nel i 577, entrò poi al servizio del duca Alfonso II e ricoprì vari incarichi anche presso il suo successore. Una volta annessa Ferrara allo Stato della Chiesa, si dedicò completamente agli studi e nel I $602 \mathrm{fu}$ tra $\mathrm{i}$ fondatori dell'Accademia degli Intrepidi. Autore di alcune rime volgari, fu però essenzialmente un traduttore e un volgarizzatore; uscì postuma, a cura del figlio Lodovico, la versione dei sei libri Della Politica, overo Del Governo di Stato di Giusto Lipsio, ma i suoi più grandi successi editoriali riguardano le opere che tradusse dal francese, tutte ristampate varie volte: L'agriculture et maison rustique di Charles Estienne (L'agricoltura di casa e di villa, Venezia: Aldo, I 58 I), De la vicissitude ou varieté des choses... di Louis Le Roy (La vicissitudine e mutabile varietà delle cose dell'universo di Luigi Regio, Venezia: Aldo, i 585 ) e la Démonomanie des sorciers di Jean Bodin (La demonomania degli stregoni, Venezia: Aldo, I 587 ).

I 3. La querelle resta viva ben aldilà del Cinquecento. Solo nel i986, con una bolla di papa Giovanni Paolo II, è stata creata la nuova diocesi di Adria-Rovigo, che include 44 comuni dell'alto e medio Polesine, con sede vescovile ormai a Rovigo - ma denominazione doppia, con Adria in primo luogo. Cfr. F. Agostini [et alii], Diocesi di Adria - Rovigo, [Giunta regionale del Veneto], Padova: Gregoriana, 200 I («Storia religiosa del Veneto», vol. 9).

I4. Cfr. G. Benzoni, Le Accademie, in Storia della cultura Veneta. Il Seicento, 4/I, Vicenza: Neri Pozza, I983, pp. I 3 I-62; M. Rinaldi, Le Accademie del Cinquecento, in Il Rinascimento italiano e l'Europa. vol. 2 Umanesimo ed educazione a cura di G. e R. Drusi, Fondazione Cassamarca, Treviso: Angelo Colla, 2007, pp. 337-59.

i s. Cfr. La prima parte de le rime di Magagnò, Menon, e Begotto in lingua rustica padovana, con una tradottione del primo canto de m. Ludovico Ariosto, In Padova: per Gratioso Perchacino, 1558. La seconda parte [...], In Venetia: appresso a Giovan Iacomo Albani, i 562 (colophon I 563). La terza parte [...], In Venetia: appresso Bolognino Zaltieri, i 569 . La quarta parte [...], In Venetia: presso Giorgio Angelieri, s.d. (ma non prima del i 583 ). Tutte ebbero varie ristampe.

I6. Nel ms. A i6 inf. della Biblioteca Ambrosiana di Milano si trovano, oltre a una lettera del Groto a Francesco Trento (cc. 1 7 r-1 8r), un Capitolo sopra il ventiduto di Fr. Trento del Maganza (cc. $27 \mathrm{r}-3 \mathrm{Iv}$ ), cui seguono le Lode di diversi date all'Eolia fabbricata da Fr. Trento e una Iscrizione sopra l'Aeolia fabricata l'anno I570 [...] nella villa di Custoza territorio di Vicenza.

17. Cfr. B. Spaggiari, «Le rime 'in lingua rustica' di Luigi Groto (il Cieco d'Adria)», nei Quaderni Veneti, 4/2(2015), pp. I-38.

I 8. PRIMO, ET SECONDO | VOLUME DELLE RIME | MORALI DI M. PIETRO | MASSOLO, GENTIL' HUOMO | VINITIANO, | Hora Don Lorenzo Monaco | Cassinese | [...] | Sonetti ottocento, con dua tavole nel fine, 
una | dell'opera \& l'altra de nomi di coloro, | a cui sono scritti i Sonetti. | In Fiorenza: Nella stamperia Ducale, Appresso i figliuoli | di M. Lorenzo Torrentino, \& Bernardo | Fabroni compagni. I 564.

19. Cfr. Le Rime, vol. I, pp. CXXXIII-CXXXIV. Curiosamente, il sonetto del Groto scompare nella successiva edizione veneziana: RIME MORALI | di M. Pietro Massolo | Gentilhuomo Vinitiano, | hora don lorenzo monaco cassinese, | Diuise in Quattro Libri; | Col Commento di M. Francesco Sansovino [...] In Venetia | Appresso Gio. Antonio Rampazzetto | MDLXXXIII. Cfr. la scheda relativa al volume nel Catalogo online della Fondation Barbier-Müller di Ginevra.

20. Le date sono compatibili, essendo nato il Massolo intorno al i 520 , cioè almeno venticinque anni prima della morte del Bembo. Quanto egli afferma non è però verificabile, tanto più che le citate opere latine non risultano pubblicate, con o senza la benedizione dell'illustre Cardinale.

2I. SONETTI MORALI | DI M. PIETRO MASSOLO | Gentilhuomo venetiano, | HORA DON LORENZO | MONACO CASSINESE | Al Reverendiss. Card. | Farnese. || In Bologna per Antonio Manvtio, nell'anno | MDLVII.

22. Nel is85, anno della morte del Groto, il Massolo partecipò ancora alla miscellanea in memoria di Leonora d'Este, organizzata da Gregorio Ducchi. Accanto a lui, ritroviamo alcuni personaggi a vario titolo legati al Cieco d'Adria, da Giovan Battista Maganza ("L'Anhelante Academico Olimpico») ad Angelo Ingegneri ("Il Negletto Academico Olimpico») e Antonio Beffa Negrini (I $532-$ I602, nobile bresciano, giurista e notaio oltre che letterato, membro di varie accademie: quella dei Pastori Frattegiani col nome di Negrino, degli Occulti di Brescia col nome di Caliginoso, e degli Innominati di Parma). Cfr. LAGRIME | DI DIVERSI POETI | VOLGARI, ET LATINI, sparse per la Morte | dell'illustriss. et eccellentiss. | MADAMA LEONORA D'ESTE. | Et raccolte da GREGORIO Dvcchr. | Et dallui dedicate all'Illustriss. \& Reuerendiss. Sig. | Cardinal d'Este suo Signore. || In VICENZA, | Nella stamperia noua, MDLXXXV.

23. Cfr. VIRIDARIUM | POETARUM | Tum Latino, tum Græco, tum Vulgari | eloquio scribentium. | In laudes | serenissimi, | atque potentissimi | D. D. STEPHANI | REGIS POLONIAE. | In duos libros divisum. Venetiis: ad signum Hyppogriphi, I 583 . Il secondo volume, con frontespizio autonomo: DEL | GIARDINO | DE' POETI, | In lode del Serenissimo | RE DI POLONIA, | Gran Duca di Littuania, Russia, Prussia, | Samogitia, e Chiouia, Signor | di Libonia | \& | PRENCIPE DI TRANSILVANIA, | LIBRO SECONDO. [ritratto in ovale del re, affiancato dalla scritta «STEFANO BATTORE $\mid$ RE DI POLONIA, \&C. »] | In VENETIA, Appresso i Guerra, fratelli, I 583.

24. Fatto di eccezionale rarità, per l'epoca in cui visse Modesta Pozzo, nonostante appartenesse al ceto superiore dei 'cittadini originari'. Cfr. D. Martelli, Polifonie. Le donne a Venezía nell'età di Moderata Fonte (seconda metà del secolo XVI), Padova, CLEUP, 20I I.

25. Bellunese di origine e notaio di professione, il Doglioni appartenne sempre al Consiglio dei nobili della città cadorina, benché nato e vissuto a Venezia, dove 
prese moglie e accolse in casa i nipoti rimasti orfani. Lasciò varie opere storiche ed erudite, in parte pubblicate e in parte ancora manoscritte, tra cui un Trattato dell'origine di Belluno (Venezia: Rampazzetto, I 588). Subito dopo la morte per parto di Modesta, avvenuta nel I 592, Doglioni si propose di dare alle stampe Il Merito delle donne, che uscì però soltanto nel I 600 per i tipi dell'Imberti. Scrisse anche la biografia di Modesta Pozzo che precede questo trattato in difesa della condizione femminile, nella quale si premura di mettere in luce le singolari doti della nipote, donna che riuscì a conciliare l' 'anomalia' dello studio e della scrittura con l'inevitabile funzione di sposa e madre esemplare (Vita della signora Modesta Pozzo di Zorzi nominata Moderata Fonte). Cfr. anche l'art. di Marina Romanello in Di\%: Biografico degli Italiani, 40 (I99I).

26. A lei fu dedicata la celebre miscellanea di rime in morte, curata dall'Attanagi, che raccolse ben 279 poesie in volgare e 102 carmi latini: RIME DI DIVERSI | Nobilissimi, et ECCEL- | Lentissimi autori, | In morte della Signora Irene | delle Signore di Spilimbergo. | Alle quali si sono aggiunti versi Latini | di diversi egregij Poeti, in morte | della medesima Signora. I In Venetia, appresso Domenico, \& Gio. | Battista Guerra, fratelli, I 56r. Per una volta, gli autori "nobilissimi et eccellentissimi" vantati nel titolo del volume rappresentano davvero quanto di meglio aveva prodotto la lirica italiana alla metà del Cinquecento. Si contano, fra gli altri, Benedetto Varchi, Bernardo Tasso e il giovane Torquato (alla sua prima pubblicazione), Bernardino Rota, Celio Magno, Curzio Gonzaga, Domenico e Marco Venier, Giovan Battista Amalteo, Lodovico Dolce, Lodovico Paterno, Luca Contile, Luigi Tansillo, Girolamo Muzio, Olimpia Malipiero e il Maganza.

27. Cfr. Del Giardino, op. cit., p. 22: Del medesimo [Gervaso Gervasi] Spirto gentil, che con purgati inchiostri; p. 23 DI LVIGI GROTTO. IN RISPOSTA. Voi, che con l'aurea penna, non gli inchiostri. Ivi, p. 39 DI ANTONIO CAVASSEGO Illustrissimo al mondo unico, e solo; DI LVIGI GROTTO. Voi sol pingete il Re Stefano solo.

28. Cfr. anche Le Rime, I, pp. cxxxvi-cxxxix.

29. Cfr. Dizionario Storico-Artistico-Letterario Bellunese compilato dal conte Florio Miari, Belluno: dalla tipografia di Francesco Deliberali, [1 843] (anast. Bologna: Forni, I 979), p. 79.

30. M. Maylender, Storia delle Accademie d'Italia, Bologna: L. Cappelli, I 926-I 930, in 5 vol., [rist. Bologna: Forni, I976], II, pp. 27-28. Ivi: «Fiorirno per longo spatio d'anni questi honorati Accademici con molt'utile et splendore di quella nobilissima et antichissima Città Bellona, o Belluno», in Accademia Colle Bellunese De' ragionamenti accademici, poetici, morali, astrologici, naturali \& varij dilettevoli, \& eruditi: del M. Illustre \& eccellente sig. Gio. Colle Bellunese de' nobili di S. Bartholameo de Colle [...]. Parte prima. In Venetia: appresso Evangelista Deuchino, i62 I: «A' benigni lettori».

3 I. Cfr. Bibliografia Bellunese compilata da Augusto Buzzati, Venezia, Tipografia dell'Ancora, L. Merlo, I 890, pp. xli-xlii. Il più noto fu Bartolomeo Cavassico (ca. I480-1555): «Nella sua attività di notaio, fra una scampagnata nei poderi che possedeva nei dintorni di Belluno e una festa carnevalesca, anche il Cavassico si dilettò nella composizione di versi il più delle volte nel rustico dialetto nativo, 
talora anche in una lingua letteraria imbellettata e chiazzata di elementi dialettali. Ma questi suoi tentativi non varcavano i limiti della sua famiglia, la cerchia ristretta degli amici, o al più, in certi casi, le mura della città, ed è molto che non sieno andati perduti». L'autografo delle sue poesie, conservato nel Museo civico di Belluno, è un manoscritto di 247 carte, con l'annotazione «I 508. Liber mei Bartholomei Cavassici not. ${ }^{\mathrm{ii}} \gg$. Cfr. Le Rime di Bartolomeo Cavassico, notaio bellunese della prima metà del secolo XVI, con introduzione e note di Vittorio Cian e con illustrazioni linguistiche e lessico a cura di Carlo Salvioni, vol. I, In Bologna, Romagnoli dell'Acqua, I 893.

32. L'orazione non è intitolata a «Luigi Dolfin Podestà e Capitano di Bennumo negli anni i 582-83", come riporta il Pagani, bensì Oratione | al clariss. Sig. Lvigi Delfino | Podestà, \& Capitanio | Di cividal di Bellvno | Gli Anni i 582 , \& I 583 ». Cfr. Marino Pagani, Catalogo ragionato delle opere dei principali scrittori bellunesi non viventi, Belluno: dalla tipografia Tissi, I844, p. 20 . Possiamo aggiungere due opuscoletti latini, di poche pagine in- $4^{\circ}$, pubblicati entrambi a Venezia: Antonius Cavassicus Bellunensis, clara academiam Venetum dum pandit in urbe, in sua vota pius numina magna vocat, Venetiis: apud Io. Antonium Rampazzetum, i 595; ANTONII Cavassici Bellunensis Verba Cylleneae fidei associanda. In electione serenissimi Venetiarum principis Marini Grimani odae tres vulgatas per artes deductae [...], Venetiis: apud Guerraeos, I 596.

33. In Vinegia: appresso Gabriel Giolito de Ferrari, I 594 [princeps: I 544 ], in due libri, dedicati rispettivamente a Bona Sforza, regina di Polonia, e alla figlia Isabella (cfr. ora l'edizione a c. di Roberto Gigliucci, Torino: RES, 2004). L'autore, Ludovico Domenichi, apparteneva come il Groto all'Accademia dei pastori Frattegiani (v. supra, p. I). Si ricordi che dal matrimonio di Bona Sforza con Sigismondo I nacque anche un figlio maschio, Sigismondo II, destinato a morire senza eredi nel I 572; con lui finì la dinastia dei Jagellonidi, aprendo così la strada all'elezione del nuovo re di Polonia, quello Stefano Báthory di cui sopra.

34. In Vinegia: appresso Gabriel Giolito de Ferrari, i 548, dedicato ad Ercole II d'Este. Per la descrizione del volume si rimanda alla scheda corrispondente nel catalogo online della Fondation Barbier-Müller di Ginevra.

35. PESCATORIA ET EC $=\mid$ LOGHE DEL SAN $\mid$ MARTINO, senza alcuna indicazione di autore, di data e luogo di stampa. Carte non numerate $\mathrm{A}_{\mathrm{I}-\mathrm{M}_{3}}$. Nel frontespizio, la marca tipografica dei Giolito con le iniziali $I G F$ iscritte nel tondo. Dovrebbe trattarsi di Giovanni Giolito de' Ferrari, il padre di Gabriele, che usava appunto come iniziale la forma latina del nome, Iohannes. Fu attivo a Trino (Vercelli), poi in Piemonte e a Venezia. Morì nel I 539-I 540 (ante quem).

36. LA PSICHE | DI HERCOLE | VDINE | Con una breve Allegoria | Del molto R. P. | D. Angelo Grillo | Dedicata | Alla Sereniss. Madama | Leonora Medici | GONZAGa. | Duchessa di Mantova, | et di Monferrato, etc. || In Venetia, Presso Gio. Battista Ciotti i 599. Poemetto in ottave, diviso in otto canti, che rielabora la favola di Apuleio (ed. a cura di Salvatore Ussia, Vercelli: Mercuri, 2004). Anche Ercole Udine apparteneva all'Accademia dei pastori Frattegiani, con lo pseudonimo Mopso. 
37. LAMENTO | DOLOROSO | DELL'ITALIA | commemorando gli huomini il- | lustri in arme, e in lette- | re in quella creati. [...]. [Marca tipografica: La Fede (donna seduta con una croce in braccio), con ai lati la scritta «senza di me l'huom | fassi a dio ribello»]. Explicit: Stampata in Vinegia per Matthio Pagano in | Frezzaria al Segno della Fede, I 559. Senza nome d'autore; i6 carte non numerate. Contiene 7 capitoli in terza rima.

38. Data l'origine virgiliana, Damon(e) era d'altronde uno pseudonimo ricorrente nelle accademie con vocazione pastorale.

39. Ancor oggi la Polonia è un'isola saldamente cattolica, nonostante si trovi in diretto contatto con popoli slavi o baltici.

40. Martelli, Polifonie, cit., nota i i6, p. 205: «Rime di Giannetta Tron [...] si trovano in L. Bergalli, Componimenti, I 726, Parte I. Cfr. M. Foscarini, Della letteratura, 1976 [1752] p. 576. Cfr. Quadrio, Della storia, 1739-52, II, I, p. 525, Cfr. J. Morelli, «Della cultura» (1796), p. 206. Cfr. G. Canonici Fachini, Prospetto, i 824. Cfr. P. L. Ferri, Biblioteca, I 842. Cfr. E. Musatti, La donna, I 892, p. I 30. Cfr. I. De Blasi, Le scrittrici, I930, p. Io6. Cfr. M. Bandini Buti, Poetesse, I942, II, p. 3 I 8.

4I. Martelli, Polifonie, cit., nota I I 5, p. 205: «Rime di Rosa L. (Rosa Levi) [...] si trovano in L. Bergalli, Componimenti, I726, Parte I. Cfr. Quadrio, Della storia, I739-52, II, I, p. 525. Cfr. G. Canonici Fachini, Prospetto, i 824. Cfr. P. L. Ferri, Biblioteca, I 842. Cfr. E. Musatti, La donna, I 892, p. I 3 I. Cfr. I. De Blasi, Le scrittrici, 1930, p. ı06. Cfr. M. Bandini Buti, Poetesse, I942, I, p. 340». Aggiungiamo che Rosa Levi ebbe un rilievo particolare nelle Rime di Luigi Groto, che scrive per lei un sonetto in morte del fratello musicista (I.207); un madrigale in cui la ringrazia di averlo ospitato nella sua casa (I.3 I I); tre distici amorosi in metro barbaro (II.283) e un sonetto per celebrare la sua conversione (III.260 D'una Hebrea, che si batterza).

42. Col titolo Lettera del filosofo Lyndorach al Re Culteber, delli incommodi de maritati, il testo - suddiviso in otto capitoli - viene inviato «Di Hadria, il dì I 4 di Decembre I 570» ad Agostino Poggi (cfr. L. Groto, Lettere Famigliari, In Venetia: Appresso Giovachino Brugnolo, I6o I, ff. 5v-I 3r). Cfr. anche Le Rime, vol. I, pp. lxxi-lxxiii.

43. Pubblicato in due diverse redazioni, prima nelle Rime piacevoli raccolte da Giacomo Cescato (In Vicenza: Per Giorgio Greco, I603, ff. I64V-I67v), poi nell'edizione delle Rime grotiane di Ambrogio Dei (i6ro: Seconda Parte, no 631). Cfr. Le Rime, vol. I. pp. cxliv-cl (il testo nel vol. II, pp. 1084-97: II. 63 I Consiglio a chi è da maritarsi).

44. Cfr. Giovanni Della Casa, Se s'abbia da prender moglie. Traduzione di Ugo Enrico Paoli, Firenze: Le Monnier, I 944.

45. «A Messer Annibale Malegucio» (Malaguzæi) che progettava le nozze con Lucrezia Pio. Cfr. Ludovico Ariosto, Opere minori, a c. di Cesare Segre, Milano-Napoli: Ricciardi, I954, pp. 548-60. 